\title{
Division algebras in linear Gr-categories
}

\author{
Hua-Lin Huang Fred Van Oystaeyen Yinhuo Zhang
}

\begin{abstract}
We study division algebras in an arbitrary linear Gr-category, i.e., a category of finite-dimensional vector spaces graded by a group with associativity constraint given by a 3-cocycle. When the 3-cocycle is non-coboundary, this provides some interesting classes of nonassociative division algebras. In particular, when we work on Gr-categories over the field of real numbers, some quasi-associative version of the quaternions and octonions appear.
\end{abstract}

\section{Introduction}

By a linear Gr-category we mean a category of finite-dimensional vector spaces graded by a group with the natural tensor product of graded vector spaces. In 1975, the monoidal structures of Gr-categories were related to group cohomology of degree 3 in the thesis of Hoàng Xuân Sính [13]. Gr-categories are probably the simplest class of monoidal categories, hence an excellent testing ground for general ideas, methods and problems in monoidal categories, quantum groups and related topics, see for instances [15, 10, 18].

The starting point of this note was our attempt to compute the Brauer group of an arbitrary braided Gr-category. As is well-known, the crux of the classical Brauer group theory of a field lies in the classification of central division algebras over the field [17]. As for the Brauer groups of braided monoidal categories, the problem in many cases naturally transfers to the classification of central division algebras in the ground categories, see [20]. This subject matter will be treated in future works.

We notice that algebras can be defined in any monoidal category without regard to its braided structures, so it is natural to extend our investigation to

Received by the editors in June 2013 - In revised form in May 2014.

Communicated by S. Caenepeel.

2010 Mathematics Subject Classification : 17A35, 20J06, 18 D10.

Key words and phrases : division algebra, group cohomology, Gr-category. 
general (not necessarily braided) Gr-categories. On the other hand, we feel that the theory of division algebras in monoidal categories should be of independent interest and it merits a separate consideration. Aside from their obvious connection to the theories of Brauer groups and module categories over monoidal categories [19], it seems also reasonable to expect that such division algebras would have similar applications in quantum (specifically, nonassociative) geometry and physics as their classical analogue quaternions, octonions and generalizations do, see e.g. $[11,9,6,7,1]$.

Throughout the note, we work over a field $\mathbb{k}$ with identity denoted by 1 . Vector spaces, algebras, morphisms, and categories are over $\mathbb{k}$ unless otherwise specified. After recalling some basic definitions concerning Gr-categories and algebras therein in Section 2, we provide in Section 3 the classification of division algebras in a Gr-category via some triples consisting of associative division $\mathbb{k}$-algebras, almost group homomorphisms and 2-cocycles of groups. In Section 4, we give a description of iso-classes of division algebras in a Gr-category mainly by some cohomological data of finite groups. Some explicit examples, in particular, some quasi-associative version of the quaternions and octonions, are given in Section 5.

\section{Linear Gr-categories}

Let $G$ be a finite group with identity element 2 . A normalized 3-cocycle $\omega$ on $G$ with coefficients in $\mathbb{k}^{*}:=\mathbb{k} \backslash\{0\}$ is a map

$$
\omega: G \times G \times G \longrightarrow \mathbb{k}^{*}
$$

such that the following equalities hold for all $e, f, g, h \in G$ :

$$
\begin{gathered}
\omega(e, f, g) \omega(e, f g, h) \omega(f, g, h)=\omega(e f, g, h) \omega(e, f, g h), \\
\omega(l, e, f)=\omega(e, l, f)=\omega(e, f, \imath)=1 .
\end{gathered}
$$

By $\mathcal{C}(G, \mathbb{k})$ we denote the category of finite-dimensional $G$-graded $\mathbb{k}$-vector spaces. The usual tensor product $\otimes$ over $\mathbb{k}$ is a bifunctor of $\mathcal{C}(G, \mathbb{k})$. Given a 3-cocycle $\omega$ on $G$, one can define an associativity constraint on $\mathcal{C}(G, \mathbb{k})$ by

$$
\begin{aligned}
& \omega_{U, V, W}:(U \otimes V) \otimes W \longrightarrow U \otimes(V \otimes W) \\
& (u \otimes v) \otimes w \mapsto \omega(|u|,|v|,|w|) u \otimes(v \otimes w),
\end{aligned}
$$

where $|v|$ denotes the degree of a homogeneous element $v \in V$. The tensor functor $\otimes$ and the associativity constraint $\omega$ provide a monoidal structure on $\mathcal{C}(G, \mathbb{k})$. This is what we called a linear Gr-category. To emphasize the associativity isomorphism, we denote the monoidal category by $\mathcal{C}(G, \omega, \mathbb{k})$. It is well-known that the monoidal structures on $\mathcal{C}(G, \mathbb{k})$ are parameterized by $\mathrm{H}^{3}\left(G, \mathbb{k}^{*}\right)$, see [13] or the more recent [8].

An algebra in $\mathcal{C}=\mathcal{C}(G, \omega, \mathbb{k})$ is an object $A$ with a multiplication morphism $m: A \otimes A \rightarrow A$ and a unit morphism $u: \mathbb{k} \rightarrow A$ satisfying the associativity and the unitary conditions of usual algebras but expressed in diagrams of the 
category $\mathcal{C}$. More precisely, an algebra in $\mathcal{C}$ is a finite-dimensional $G$-graded space $A=\oplus_{g \in G} A_{g}$ with a multiplication $\cdot$ such that

$$
\begin{gathered}
A_{g} \cdot A_{h} \subseteq A_{g h} \\
(a \cdot b) \cdot c=\omega(|a|,|b|,|c|) a \cdot(b \cdot c)
\end{gathered}
$$

for all homogeneous elements $a, b, c \in A$. There is also a unit element $\mathbf{1}$ in $A$ such that

$$
\mathbf{1} \cdot a=a=a \cdot \mathbf{1}
$$

for all $a \in A$. Apparently, $\mathbf{1} \in A_{1}$.

Algebras in linear Gr-categories were studied in [2], where they were called group graded quasialgebras. These algebras form an interesting class of nonassociative, but almost associative up to a 3-cocycle, algebras including the wellknown quaternions, octonions and higher Cayley algebras, see loc. cit. When the group is $\mathbb{Z}_{2}$, or more generally a finite abelian group, some classification results of simple algebras in the corresponding Gr-categories were obtained in [3,4].

\section{Division algebras in Gr-categories}

Let $\mathcal{C}=\mathcal{C}(G, \omega, \mathbb{k})$ be a Gr-category. An algebra $A$ in $\mathcal{C}$ is said to be a division algebra if any nonzero homogeneous element has a left and a right inverse. A division algebra $A$ in $\mathcal{C}$ is said to be ample, if the homogeneous spaces $A_{g}$ are nonzero for all $g \in G$. Obviously it is enough to study ample division algebras in $\mathcal{C}$ as non-ample ones will become ample in a Gr-category with smaller group and the restriction of a 3-cocycle is again 3-cocycle.

Lemma 3.1. Let $A=\oplus_{g \in G} A_{g}$ be an ample division algebra in $\mathcal{C}$.

1. $A_{l}$ is an associative division $\mathbb{k}$-algebra.

2. $A_{\imath} a_{g}=A_{g}=a_{g} A_{\imath}$ for any $a_{g} \in A_{g} \backslash\{0\}$.

3. If $a_{g} \in A_{g} \backslash\{0\}$, then $\exists a_{g}^{l}, a_{g}^{r} \in A_{g-1}$ such that $a_{g}^{l} a_{g}=\mathbf{1}=a_{g} a_{g}^{r}$. Moreover, $a_{g}^{r}=\omega\left(g^{-1}, g, g^{-1}\right) a_{g}^{l}$.

4. If $a_{g} \in A_{g} \backslash\{0\}$, then $\phi_{g}: A_{l} \rightarrow A_{l}, d \mapsto a_{g} d a_{g}^{r}$ is an associative $\mathbb{k}$-algebra automorphism.

Proof. Trivial.

Remark 3.2. For an ample division algebra $A$ in $\mathcal{C}$, one can choose a set of elements $\left\{a_{g} \mid g \in G\right\}$ with $a_{\imath}=\mathbf{1}$ and $a_{g} \in A_{g} \backslash\{0\}$ such that $A=\oplus_{g \in G} A_{\imath} a_{g}$.

Let $A$ be an ample division algebra in $\mathcal{C}$ as above. From now on, we fix a set $\left\{a_{g} \in A_{g} \mid g \in G\right\}$ of homogeneous elements of $A$ as in Remark 3.2. Denote by $D$ the associative division $\mathbb{k}$-algebra $A_{l}$. We have the associated map

$$
\phi: G \rightarrow \operatorname{Aut}(D), \quad g \mapsto \phi_{g} .
$$


Note that $a_{g} a_{h} \in A_{g h}$. Hence there is a unique element $\sigma(g, h)$ in $D^{*}:=D \backslash\{0\}$ such that $a_{g} a_{h}=\sigma(g, h) a_{g h}$. In particular, $\sigma$ is normalized:

$$
\sigma(l, g)=1=\sigma(g, \imath), \forall g \in G \text {. }
$$

This results in a 2-cochain on $G$ with coefficients in the division algebra $D$ :

$$
G \times G \stackrel{\sigma}{\rightarrow} D^{*}, \quad(g, h) \mapsto \sigma(g, h) .
$$

For each $c \in D^{*}$, define

$$
\operatorname{Ad}(c): D \rightarrow D, \quad d \mapsto c d c^{-1}
$$

Clearly $\operatorname{Ad}(D)$ is the group $\operatorname{Inn}(D)$ of inner automorphisms of the division kk-algebra $D$.

Lemma 3.3. Keep the above conventions. We have

$$
\begin{gathered}
\sigma(f, g) \sigma(f g, h)=\omega(f, g, h) \phi_{f}(\sigma(g, h)) \sigma(f, g h), \\
\phi_{f} \circ \phi_{g}=\operatorname{Ad}(\sigma(f, g)) \circ \phi_{f g}
\end{gathered}
$$

for all $f, g, h \in G$.

Proof. By straightforward computations, (3.1) is derived from

$$
\left(a_{f} \cdot a_{g}\right) \cdot a_{h}=\omega(f, g, h) a_{f} \cdot\left(a_{g} \cdot a_{h}\right),
$$

while (3.2) is from the definitions of $\phi, \sigma$ and Lemma 3.1.

So far we have observed the main ingredients of an ample division algebra in $\mathcal{C}$, namely, an associative division $\mathbb{k}$-algebra $D$, an almost group homomorphism $\phi: G \rightarrow \operatorname{Aut}(D)$ and a normalized 2-cochain $\sigma: G \times G \rightarrow D^{*}$ satisfying (3.1)(3.2). From now on, we use $(D, \phi, \sigma)$ to denote these data and call it a good triple in $\mathcal{C}$.

Conversely, given a good triple $(D, \phi, \sigma)$ as above, we can construct an ample division algebra in $\mathcal{C}$ as follows. Regard $A=\oplus_{g \in G} D a_{g}$ as a $G$-graded space with $A_{g}=D a_{g}$. Take $a_{\imath}=1$, the unit of $D$, while view $a_{g}$ with $g \neq \imath$ as a symbol. Define a multiplication on $A$ by

$$
\left(x a_{g}\right) \cdot\left(y a_{h}\right)=x \phi_{g}(y) \sigma(g, h) a_{g h}
$$

for all $x, y \in D$ and $g, h \in G$.

Lemma 3.4. The so-defined algebra $A$ associated to the triple $(D, \phi, \sigma)$ is an ample division algebra in $\mathcal{C}$.

Proof. The product (3.3) is $\mathbb{k}$-bilinear and satisfies (2.4) by definition. Take the unit $\mathbf{1}_{A}$ of $A$ as the unit $\mathbf{1}$ of $D$. Clearly it satisfies (2.6). Finally, (2.5) follows from (3.1)-(3.2): for all $f, g, h \in G, x, y, z \in D$ we have:

$$
\begin{aligned}
{\left[\left(x a_{f}\right)\left(y a_{g}\right)\right]\left(z a_{h}\right) } & =\left(x \phi_{f}(y) \sigma(f, g) a_{f g}\right)\left(z a_{h}\right) \\
& =x \phi_{f}(y) \sigma(f, g) \phi_{f g}(z) \sigma(f g, h) a_{f g h} \\
& =x \phi_{f}(y) \operatorname{Ad}(\sigma(f, g)) \circ \phi_{f g}(z) \sigma(f, g) \sigma(f g, h) a_{f g h} \\
b y(3.1)-(3.2) & =x \phi_{f}(y) \phi_{f} \circ \phi_{g}(z) \omega(f, g, h) \phi_{f}(\sigma(g, h)) \sigma(f, g h) a_{f g h} \\
& =\omega(f, g, h) x \phi_{f}\left(y \phi_{g}(z) \sigma(g, h)\right) \sigma(f, g h) a_{f g h} \\
& =\omega(f, g, h)\left(x a_{f}\right)\left[\left(y a_{g}\right)\left(z a_{h}\right)\right] .
\end{aligned}
$$


To summarize, we have

Proposition 3.5. In a $\mathrm{Gr}$-category $\mathcal{C}$, ample division algebras are in 1-1 correspondence with good triples.

\section{Isomorphism classes}

In this section, we classify the isomorphism classes of ample division algebras in $\mathcal{C}=\mathcal{C}(G, \omega, \mathbb{k})$ by means of good triple data. Let $A$ and $A^{\prime}$ be two ample division algebras in $\mathcal{C}$. If there is an invertible morphism $F: A \rightarrow A^{\prime}$ in $\mathcal{C}$ which satisfies

$$
F\left(\mathbf{1}_{A}\right)=\mathbf{1}_{A^{\prime}}, \quad F\left(a \cdot{ }_{A} b\right)=F(a) \cdot A^{\prime} F(b), \forall a, b \in A,
$$

then we say $A$ and $A^{\prime}$ are isomorphic as ample division algebras in $\mathcal{C}$. For convenience, we will also say that the corresponding good triples of $A$ and $A^{\prime}$ are isomorphic according to Proposition 3.5. From now on we always write $A=$ $\oplus_{g \in G} D a_{g}$ and $A^{\prime}=\oplus_{g \in G} D^{\prime} a_{g}^{\prime}$, and their corresponding good triples as $(D, \phi, \sigma)$ and $\left(D^{\prime}, \phi^{\prime}, \sigma^{\prime}\right)$ respectively.

Lemma 4.1. Assume that $F: A \rightarrow A^{\prime}$ is an isomorphism of ample division algebras in C. Then

1. $\alpha=\left.F\right|_{D}: D \rightarrow D^{\prime}$ is an isomorphism of associative division $\mathbb{k}$-algebras, and

2. there exists a set of nonzero elements $\left\{d_{g}^{\prime}\right\}_{g \in G}$ in $D^{\prime}$ such that

$$
\begin{gathered}
d_{\imath}^{\prime}=\mathbf{1}_{A^{\prime}} \\
\alpha\left(\phi_{g}(y)\right) \alpha(\sigma(g, h)) d_{g h}^{\prime}=d_{g}^{\prime} \phi_{g}^{\prime}\left(\alpha(y) d_{h}^{\prime}\right) \sigma^{\prime}(g, h), \quad \forall y \in D, g, h \in G .
\end{gathered}
$$

Conversely, given an isomorphism $\alpha: D \rightarrow D^{\prime}$ of division $\mathbb{k}$-algebras and a set of elements $\left\{d_{g}^{\prime} \in D^{\prime *} \mid g \in G\right\}$ satisfying (4.2), there is an isomorphism of ample division algebras in $\mathcal{C}$ given by

$$
F: A \rightarrow A^{\prime}, \quad x a_{g} \mapsto \alpha(x) d_{g}^{\prime} a_{g}^{\prime}, \quad \forall x \in D, g \in G
$$

Proof. Assume that $F: A \rightarrow A^{\prime}$ is an isomorphism. The claim (1) is obvious by definition. For (2), note that $F$ preserves degree, hence $F\left(a_{g}\right)=d_{g}^{\prime} a_{g}^{\prime}$ for some $d_{g}^{\prime} \in D^{\prime *}$. Then (4.2) is a direct consequence of (4.1) by letting $a=x a_{g}, b=$ $y a_{h}, \forall x, y \in D, g, h \in G$.

The converse follows a direct verification of (4.1), which is clearly guaranteed by (4.2) and goes as follows:

$$
\begin{aligned}
F\left(x a_{g} \cdot A_{A} y a_{h}\right) & =F\left(x \phi_{g}(y) \sigma(g, h) a_{g h}\right) \\
& =\alpha\left(x \phi_{g}(y) \sigma(g, h)\right) d_{g h}^{\prime} a_{g h}^{\prime} \\
b y(4.2) & =\alpha(x) d_{g}^{\prime} \phi_{g}^{\prime}\left(\alpha(y) d_{h}^{\prime}\right) \sigma^{\prime}(g, h) a_{g h}^{\prime} \\
& =\left[\alpha(x) d_{g}^{\prime} a_{g}^{\prime}\right] \cdot A^{\prime}\left[\alpha(y) d_{h}^{\prime} a_{h}^{\prime}\right] \\
& =F\left(x a_{g}\right) \cdot A^{\prime} F\left(y a_{h}\right)
\end{aligned}
$$

for all $x, y \in D, g, h \in G$. 
Let $(D, \phi, \sigma)$ be a good triple. Following Lemma 4.1, a division algebra isomorphism $\alpha: D \rightarrow D^{\prime}$ can induce a good triple datum on $D^{\prime}$ so that the isomorphism $\alpha$ can be extended to an isomorphism of ample division algebras.

Corollary 4.2. Let $A$ be the ample division algebra in $\mathcal{C}$ corresponding to a good triple $(D, \phi, \sigma)$. Suppose that $\alpha: D \rightarrow D^{\prime}$ is an isomorphism of division $\mathbb{k}$-algebras. Let $\phi_{g}^{\prime}=\alpha \circ \phi_{g} \circ \alpha^{-1}, \forall g \in G$ and $\sigma^{\prime}=\alpha \circ \sigma$. Then the triple $\left(D^{\prime}, \phi^{\prime}, \sigma^{\prime}\right)$ is good and the associated ample division algebra $A^{\prime}$ is isomorphic to $A$ in $\mathcal{C}$ with an isomorphism $F: A \rightarrow A^{\prime}$ satisfying $\left.F\right|_{D}=\alpha$.

Proof. By assumption, one has for all $f, g, h \in G$,

$$
\begin{aligned}
\sigma^{\prime}(f, g) \sigma^{\prime}(f g, h) & =\alpha(\sigma(f, g) \sigma(f g, h)) \\
b y(3.1) & =\alpha\left(\omega(f, g, h) \phi_{f}(\sigma(g, h)) \sigma(f, g h)\right) \\
& =\omega(f, g, h) \alpha \circ \phi_{f} \circ \alpha^{-1}(\alpha \circ \sigma(g, h)) \alpha \circ \sigma(f, g h) \\
& =\omega(f, g, h) \phi_{f}^{\prime}\left(\sigma^{\prime}(g, h)\right) \sigma^{\prime}(f, g h), \text { and } \\
\phi_{f}^{\prime} \circ \phi_{g}^{\prime} & =\alpha \circ \phi_{f} \circ \alpha^{-1} \circ \alpha \circ \phi_{g} \circ \alpha^{-1} \\
b y(3.2) & =\alpha \circ \operatorname{Ad}(\sigma(f, g)) \circ \phi_{f g} \circ \alpha^{-1} \\
& =\alpha \circ \operatorname{Ad}(\sigma(f, g)) \circ \alpha^{-1} \circ \phi_{f g}^{\prime} \\
& =\operatorname{Ad}(\alpha \circ \sigma(f, g)) \circ \phi_{f g}^{\prime} \\
& =\operatorname{Ad}\left(\sigma^{\prime}(f, g)\right) \circ \phi_{f g}^{\prime} .
\end{aligned}
$$

Therefore, $\left(D^{\prime}, \phi^{\prime}, \sigma^{\prime}\right)$ is a good triple and hence there is a corresponding ample division algebra $A^{\prime}$ in $\mathcal{C}$ by Proposition 3.5.

To extend $\alpha$ to an isomorphism $F$ as in (4.3), we let $d_{g}^{\prime}=\mathbf{1}_{D^{\prime}}, \forall g \in G$. Then by the assumption we have:

$$
\begin{aligned}
\alpha\left(\phi_{g}(y)\right) \alpha(\sigma(g, h)) d_{g h}^{\prime} & =\alpha \circ \phi_{g} \circ \alpha^{-1}(\alpha(y)) \alpha \circ \sigma(g, h) \mathbf{1}_{D^{\prime}} \\
& =\mathbf{1}_{D^{\prime}} \phi_{g}^{\prime}\left(\alpha(y) \mathbf{1}_{D^{\prime}}\right) \sigma^{\prime}(g, h) \\
& =d_{g}^{\prime} \phi_{g}^{\prime}\left(\alpha(y) d_{h}^{\prime}\right) \sigma^{\prime}(g, h)
\end{aligned}
$$

for all $y \in D, g, h \in G$. Hence, $F$ is the desired isomorphism according to Lemma 4.1 .

In the following, we wish to give a complete list of iso-classes of ample division algebras in $\mathcal{C}$ by imposing appropriate restrictions on the good triples $(D, \phi, \sigma)$. First of all, by Corollary 4.2 we can fix $D$ for every good triple $(D, \phi, \sigma)$ as any division $\mathbb{k}$-algebra isomorphic to $D$ possesses a good triple isomorphic to $(D, \phi, \sigma)$. With $D$ fixed, we then look for the conditions imposed on the map $\phi: G \rightarrow \operatorname{Aut}(D)$.

Assume that a homomorphism $\phi^{\prime}: G \rightarrow \operatorname{Aut}(D)$ is given by $\phi_{g}^{\prime}=\operatorname{Ad}\left(c_{g}\right) \circ \phi_{g}, \forall g \in G$, where $c_{g} \in D^{*}$ and $\operatorname{Ad}\left(c_{g}\right) \in \operatorname{Inn}(D)$. We set $c_{l}$ to be $\mathbf{1}_{D}$ so that $\phi_{1}^{\prime}=\operatorname{Id}_{D}$. Suppose that there is a map $\sigma: G \times G \rightarrow D^{*}$ such that $(D, \phi, \sigma)$ is a good triple. Define $\sigma^{\prime}: G \times G \rightarrow D^{*}$ by

$$
\sigma^{\prime}(g, h)=c_{g} \phi_{g}\left(c_{h}\right) \sigma(g, h) c_{g h}^{-1}, \quad \forall g, h \in G
$$


Lemma 4.3. The triple $\left(D, \phi^{\prime}, \sigma^{\prime}\right)$ is good and it is isomorphic to $(D, \phi, \sigma)$.

Proof. By direct verification, one has

$$
\begin{aligned}
\sigma^{\prime}(f, g) \sigma^{\prime}(f g, h) & =c_{f} \phi_{f}\left(c_{g}\right) \sigma(f, g) c_{f g}^{-1} c_{f g} \phi_{f g}\left(c_{h}\right) \sigma(f g, h) c_{f g h}^{-1} \\
& =c_{f} \phi_{f}\left(c_{g}\right) \operatorname{Ad}(\sigma(f, g)) \circ \phi_{f g}\left(c_{h}\right) \sigma(f, g) \sigma(f g, h) c_{f g h}^{-1} \\
b y(3.1)-(3.2) & =c_{f} \phi_{f}\left(c_{g}\right) \phi_{f} \circ \phi_{g}\left(c_{h}\right) \omega(f, g, h) \phi_{f}(\sigma(g, h)) \sigma(f, g h) c_{f g h}^{-1} \\
& =\omega(f, g, h) \operatorname{Ad}\left(c_{f}\right) \circ \phi_{f}\left(c_{g} \phi_{g}\left(c_{h}\right) \sigma(g, h)\right) c_{f} \sigma(f, g h) c_{f g h}^{-1} \\
b y(4.4) & =\omega(f, g, h) \phi_{f}^{\prime}\left(\sigma^{\prime}(g, h)\right) \phi_{f}^{\prime}\left(c_{g h}\right) c_{f} \sigma(f, g h) c_{f g h}^{-1} \\
& =\omega(f, g, h) \phi_{f}^{\prime}\left(\sigma^{\prime}(g, h)\right) c_{f} \phi_{f}\left(c_{g h}\right) \sigma(f, g h) c_{f g h}^{-1} \\
b y(4.4) & =\omega(f, g, h) \phi_{f}^{\prime}\left(\sigma^{\prime}(g, h)\right) \sigma^{\prime}(f, g h), \text { and } \\
\phi_{f}^{\prime} \circ \phi_{g}^{\prime}(d) & =\operatorname{Ad}\left(c_{f}\right) \circ \phi_{f} \circ \operatorname{Ad}\left(c_{g}\right) \circ \phi_{g}(d) \\
& =c_{f} \phi_{f}\left(c_{g}\right) \phi_{f} \circ \phi_{g}(d) \phi_{f}\left(c_{g}^{-1}\right) c_{f}^{-1} \\
b y(3.2) & =c_{f} \phi_{f}\left(c_{g}\right) \operatorname{Ad}(\sigma(f, g)) \circ \phi_{f g}(d) \phi_{f}\left(c_{g}^{-1}\right) c_{f}^{-1} \\
& =c_{f} \phi_{f}\left(c_{g}\right) \sigma(f, g) \phi_{f g}(d) \sigma(f, g)^{-1} \phi_{f}\left(c_{g}^{-1}\right) c_{f}^{-1} \\
& =c_{f} \phi_{f}\left(c_{g}\right) \sigma(f, g) c_{f g}^{-1} \phi_{f g}^{\prime}(d) c_{f g} \sigma(f, g)^{-1} \phi_{f}\left(c_{g}^{-1}\right) c_{f}^{-1} \\
b y(4.4) & =\operatorname{Ad}\left(\sigma^{\prime}(f, g)\right) \circ \phi_{f g}^{\prime}(d)
\end{aligned}
$$

for all $d \in D, f, g, h \in G$. That is, the triple $\left(D, \phi^{\prime}, \sigma^{\prime}\right)$ is good.

Let $A=\oplus_{g \in G} D a_{g}$ and $A^{\prime}=\oplus_{g \in G} D a_{g}^{\prime}$ denote the associated ample division algebras to $(D, \phi, \sigma)$ and $\left(D, \phi^{\prime}, \sigma^{\prime}\right)$ respectively. Define $F: A \rightarrow A^{\prime}$ by letting $\left.F\right|_{D}=\operatorname{Id}_{D}$ and $F\left(a_{g}\right)=c_{g}^{-1} a_{g}^{\prime}, \forall g \in G$. Then for all $y \in D, g, h \in G$ we have

$$
\begin{aligned}
c_{g}^{-1} \phi_{g}^{\prime}\left(\alpha(y) c_{h}^{-1}\right) \sigma^{\prime}(g, h) & =c_{g}^{-1} c_{g} \phi_{g}\left(y c_{h}^{-1}\right) c_{g}^{-1} c_{g} \phi_{g}\left(c_{h}\right) \sigma(g, h) c_{g h}^{-1} \\
& =\phi_{g}(y) \sigma(g, h) c_{g h}^{-1} \\
u s e^{\prime \prime} \alpha=\mathrm{Id} \text { " } & =\alpha\left(\phi_{g}(y)\right) \alpha(\sigma(g, h)) c_{g h}^{-1} .
\end{aligned}
$$

Now (4.2) of Lemma 4.1 holds, so $F$ is an isomorphism.

Consider $\operatorname{Out}(D):=\operatorname{Aut}(D) / \operatorname{Inn}(D)$, the group of outer automorphisms of an associative division $\mathbb{k}$-algebra $D$. Let

$$
\pi: \operatorname{Aut}(D) \rightarrow \operatorname{Out}(D), \quad \alpha \mapsto \bar{\alpha}
$$

denote the canonical map. Note that the almost group map $\phi$ in a good triple $(D, \phi, \sigma)$ becomes a group homomorphism $\bar{\phi}=\pi \circ \phi: G \rightarrow \operatorname{Out}(D)$ thanks to (3.2).The previous lemma indicates that we need only to take one representative $\phi: G \rightarrow \operatorname{Aut}(D)$ of the induced group homomorphism $\bar{\phi}$. So, there is no harm to understand the map $\phi$ in a good triple $(D, \phi, \sigma)$ as a group homomorphism from $G$ to $\operatorname{Out}(D)$.

Two almost group homomorphisms $\phi, \phi^{\prime}: G \rightarrow \operatorname{Aut}(D)$ are said to be conjugate if there exists $\tau \in \operatorname{Aut}(D)$ such that $\overline{\phi_{g}^{\prime}}=\bar{\tau} \circ \overline{\phi_{g}} \circ \overline{\tau^{-1}}, \forall g \in G$. We denote 
the set of conjugacy classes by $C(G, \operatorname{Aut}(D))$. Furthermore, we show that $\phi$ in $(D, \phi, \sigma)$ can be even restricted to just one representative of each conjugacy class.

Lemma 4.4. Suppose that $(D, \phi, \sigma)$ is a good triple, and $\phi^{\prime}: G \rightarrow \operatorname{Aut}(D)$ an almost group homomorphism satisfying $\overline{\phi_{g}^{\prime}}=\bar{\tau} \circ \overline{\phi_{g}} \circ \overline{\tau^{-1}}, \forall g \in G$ for some $\tau \in \operatorname{Aut}(D)$. Then there exists $\sigma^{\prime} \in \operatorname{Aut}(D)$ such that $\left(D, \phi^{\prime}, \sigma^{\prime}\right)$ is a good triple and is isomorphic to $(D, \phi, \sigma)$. Conversely, if the good triples $(D, \phi, \sigma)$ and $\left(D, \phi^{\prime}, \sigma^{\prime}\right)$ are isomorphic, then $\phi$ and $\phi^{\prime}$ are conjugate.

Proof. By assumption, there is a set $\left\{c_{g} \in D^{*} \mid g \in G\right\}$ such that $\operatorname{Ad}\left(c_{g}\right) \circ \phi_{g}^{\prime}=$ $\tau \circ \phi_{g} \circ \tau^{-1}, \forall g \in G$. By Lemma 4.3, we can assume that $c_{g}=\mathbf{1}_{D}, \forall g \in G$ without loss of generality. Now let $\sigma^{\prime}=\tau \circ \sigma$, then we have

$$
\begin{aligned}
\sigma^{\prime}(f, g) \sigma^{\prime}(f g, h) & =\tau(\sigma(f, g) \sigma(f g, h)) \\
b y(3.1) & =\omega(f, g, h) \tau\left(\phi_{f}(\sigma(g, h)) \sigma(f, g h)\right) \\
& =\omega(f, g, h) A d(\tau) \circ \phi_{f}(\tau \circ \sigma(g, h)) \tau \circ \sigma(f, g h) \\
& =\omega(f, g, h) \phi_{f}^{\prime}\left(\sigma^{\prime}(f, g)\right) \sigma^{\prime}(f, g h), \text { and } \\
\phi_{f}^{\prime} \circ \phi_{g}^{\prime} & =\operatorname{Ad}(\tau) \circ\left(\phi_{f} \circ \phi_{g}\right) \\
b y(3.2) & =\operatorname{Ad}(\tau) \circ\left(\operatorname{Ad}(\sigma(f, g)) \circ \phi_{f g}\right) \\
& =\operatorname{Ad}(\tau \circ \sigma(f, g)) \circ \operatorname{Ad}(\tau) \circ \phi_{f g} \\
& =\operatorname{Ad}\left(\sigma^{\prime}(f, g)\right) \circ \phi_{f g}^{\prime}
\end{aligned}
$$

for all $f, g, h \in G$. This proves that $\left(D, \phi^{\prime}, \sigma^{\prime}\right)$ is a good triple.

Now let $A=\bigoplus_{g \in G} D a_{g}$ and $A^{\prime}=\bigoplus_{g \in G} D a_{g}^{\prime}$ denote respectively the associated ample division algebras of $(D, \phi, \sigma)$ and $\left(D, \phi^{\prime}, \sigma^{\prime}\right)$. Define $F: A \rightarrow A^{\prime}$ by letting $\left.F\right|_{D}=\tau$ and $F\left(a_{g}\right)=a_{g}^{\prime}, \forall g \in G$. The rest of the proof is a routine verification of (4.2). For all $f, g, h \in G$, one has

$$
\begin{aligned}
\tau\left(\phi_{g}(y)\right) \tau(\sigma(g, h)) & =\tau \circ \phi_{g} \circ \tau^{-1}(\tau(y)) \tau \circ \sigma(g, h) \\
& =\phi_{g}^{\prime}(\tau(y)) \sigma^{\prime}(g, h) .
\end{aligned}
$$

This proves that $F$ is an isomorphism.

For the last claim, note that there exist $\alpha \in \operatorname{Aut}(D)$ and a set $\left\{d_{g} \in D^{*} \mid g \in G\right\}$ such that (4.2) holds by Lemma 4.1. By letting $h=\imath$, the equality of (4.2) becomes

$$
\alpha\left(\phi_{g}(y)\right) d_{g}=d_{g} \phi_{g}^{\prime}(\alpha(y)), \quad \forall y \in D, \forall g \in G
$$

This says that $\operatorname{Ad}\left(d_{g}\right) \circ \phi_{g}^{\prime}=\alpha \circ \phi_{g} \circ \alpha^{-1}, \forall g \in G$. That is, $\overline{\phi_{g}}=\bar{\alpha} \circ \overline{\phi_{g}} \circ \overline{\alpha^{-1}}$, hence $\phi$ and $\phi^{\prime}$ are conjugate.

With $D$ fixed in an iso-class and $\phi$ in a conjugacy class, we are now able to work out the restrictions on $\sigma$.

Lemma 4.5. Two good triples $(D, \phi, \sigma)$ and $\left(D, \phi, \sigma^{\prime}\right)$ are isomorphic if and only if there exist $\alpha \in \operatorname{Aut}(D)$ and a set $\left\{d_{g}\right\}_{g \in G}$ in $D^{*}$ such that

$$
\begin{gathered}
\alpha \circ \phi_{g} \circ \alpha^{-1}=\operatorname{Ad}\left(d_{g}\right) \circ \phi_{g}, \quad \forall g \in G, \\
\sigma^{\prime}(g, h)=\phi_{g}\left(d_{h}^{-1}\right) d_{g}^{-1} \alpha \circ \sigma(g, h) d_{g h}, \quad \forall g, h \in G .
\end{gathered}
$$


Proof. Follows from Lemmas 4.1 and 4.4.

Let $\Sigma(D, \phi)$ denote the set of $\sigma^{\prime}$ s such that $(D, \phi, \sigma)$ forms a good triple. We remark that the set $\Sigma(D, \phi)$ might be empty. Denote by $\operatorname{Aut}(D, \phi)$ the centralizer subgroup of the subgroup $\left\{\overline{\phi_{g}} \mid g \in G\right\}$ of $\operatorname{Out}(D)$, namely,

$$
\operatorname{Aut}(D, \phi)=\left\{\alpha \in \operatorname{Out}(D) \mid \alpha \circ \overline{\phi_{g}}=\overline{\phi_{g}} \circ \alpha, \forall g \in G\right\} \text {. }
$$

Lemma 4.5 says that the group $\operatorname{Aut}(D, \phi)$ acts on the set $\Sigma(D, \phi)$ by (4.5). Now, we are in the position to state the main result of this section.

Theorem 4.6. The isomorphism classes of ample division algebras in $\mathcal{C}(G, \omega, \mathbb{k})$ are in 1-1 correspondence with the good triples $(D, \phi, \sigma)$ where $D$ represents an iso-class of associative division $\mathbb{k}$-algebras, $\phi: G \rightarrow \operatorname{Aut}(D)$ represents a conjugacy class in $C(G, \operatorname{Out}(D))$, and $\sigma: G \times G \rightarrow D^{*}$ represents an $\operatorname{Aut}(D, \phi)$-orbit of $\Sigma(D, \phi)$.

\section{Examples}

We have reduced the construction and the classification of ample division algebras in a Gr-category to some good triples. Now we apply this idea to give explicit classification results for some concrete finite groups and 3-cocycles.

Case 1. The field $\mathbb{k}_{k}$ is algebraically closed. If $D$ is a finite-dimensional associative division algebra over $\mathbb{k}$, then $D=\mathbb{k}$, and $\operatorname{Aut}(D)=\operatorname{Out}(D)=\left\{\operatorname{Id}_{\mathbb{k}}\right\}$. Let $G$ be a finite group, and $\omega$ a 3-cocycle on $G$. Ample division algebras in $\mathcal{C}(G, \omega, \mathbb{k})$ are in 1-1 correspondence with the maps $\sigma: G \times G \rightarrow \mathbb{k}^{*}$ such that $\omega=\partial \sigma$, where $\partial$ denotes the differential map. If $\omega$ is not a coboundary, then there is no ample division algebra in $\mathcal{C}(G, \omega, \mathbb{k})$. If $\omega$ is a coboundary, then $\sigma$ can be chosen in the normalized 2-cochains such that $\omega=\partial \sigma$. According to Proposition 4.6, the iso-classes of ample division algebras in $\mathcal{C}(G, \omega, \mathbb{k})$ are in 1-1 correspondence to triples $(\mathbb{k}, \mathrm{Id}, \sigma)$, where $\mathrm{Id}: G \rightarrow\left\{\mathrm{Id}_{\mathbb{k}}\right\}, g \mapsto \mathrm{Id}_{\mathbb{k}}, \forall g \in G$ and $\sigma$ represents a second cohomology class by Lemma 4.5. Therefore, the iso-classes of ample division algebras in $\mathcal{C}(G, \omega, \mathbb{k})$ are parametrized by the second cohomology group $\mathrm{H}^{2}\left(G, \mathbb{k}^{*}\right)$.

Case 2. The field $\mathbb{k}_{k}$ is $\mathbb{R}$, the real numbers. By the well-known theorem of Frobenius, there are 3 iso-classes of finite-dimensional associative division algebras over $\mathbb{R}$, namely, $\mathbb{R}$ itself, complex numbers $\mathbb{C}$, and quaternions $\mathbb{H}$. Take a finite group $G$ and a 3-cocycle $\omega$ on $G$ with coefficients in $\mathbb{R}^{*}$.

(i) $D=\mathbb{R}$, or $\mathbb{H}$. By the well-known theorem of Noether-Skolem, $\operatorname{Aut}(D)=\operatorname{Inn}(D)$. Hence there is only one choice for group homomorphisms $G \rightarrow \operatorname{Out}(D)=\left\{\operatorname{Id}_{D}\right\}$. Then we can take

$$
\phi: G \rightarrow \operatorname{Aut}(D), \quad g \mapsto \operatorname{Id}_{D}, \forall g \in G
$$

By (3.2), for all $f, g \in G$, the elements $\sigma(f, g) \in D$ should lie in the center of $D$, that is $\mathbb{R}$, and by (3.1) $\sigma$ should satisfy $\omega=\partial \sigma$. Again, if $\omega$ is not a coboundary, then there is no ample division algebra in $\mathcal{C}(G, \omega, \mathbb{R})$; if $\omega$ is a coboundary, then the iso-classes of ample division algebras in $\mathcal{C}(G, \omega, \mathbb{R})$ with $D=\mathbb{R}$ or $\mathbb{H}$ are parametrized by the second cohomology group $\mathrm{H}^{2}\left(G, \mathbb{R}^{*}\right)$. 
(ii) $D=\mathbb{C}$. In this case, $\operatorname{Aut}(\mathbb{C})=\operatorname{Out}(\mathbb{C})=\{\operatorname{Id}, \kappa\}$, where $\kappa$ is the complex conjugation. If $\phi: G \rightarrow \operatorname{Aut}(\mathbb{C}), g \mapsto$ Id is the trivial homomorphism, then we are in a similar situation as (i). If $\omega$ is not a coboundary, then there is no ample division algebra in $\mathcal{C}(G, \omega, \mathbb{R})$. If $\omega$ is a coboundary, say $\omega=\partial \zeta$, then (3.1) becomes $\partial \sigma=\partial \varsigma$ and we have $\sigma \varsigma^{-1} \in Z^{2}\left(G, \mathbb{C}^{*}\right)$, the group of 2-cocycles. By Lemma 4.5, the iso-classes of ample division algebras in $\mathcal{C}(G, \omega, \mathbb{R})$ with $D=\mathbb{C}$ are in 1-1 correspondence with the cohomology classes of $\sigma$, hence are parametrized by the second cohomology group $\mathrm{H}^{2}\left(G, \mathbb{C}^{*}\right)$.

Now assume that $\phi: G \rightarrow \operatorname{Aut}(\mathbb{C})$ is a group homomorphism such that $\phi(G)=\{\mathrm{Id}, \kappa\}$. This is possible whenever $G$ is an abelian group of even order. As a coboundary on $G$ will essentially lead us to the associative case treated in [16], we only consider non-coboundary 3-cocycles on $G$.

In order to get explicit classification results, some concrete groups must be chosen. In the following, we are mainly concerned about some possible nonassociative analogues of the quaternions and octonions. For this purpose, it is natural to consider the groups $\mathbb{Z}_{2}, \mathbb{Z}_{2 n}, \mathbb{Z}_{2}^{n}$ (with $n$ small) which are possible gradation groups for the quaternions and octonions, see e.g. $[2,12]$.

Proposition 5.1. Assume that $G=\mathbb{Z}_{2}=\left\langle g \mid g^{2}=\imath\right\rangle$. Let $\omega$ be the only non-coboundary 3-cocycle, i.e., $\omega(g, g, g)=-1$. Then there is only one, up to isomorphism, ample division algebra in $\mathcal{C}\left(\mathbb{Z}_{2}, \omega, \mathbb{R}\right)$ which is an nonassociative analogue of the quaternions.

Proof. Since $\omega$ is not a coboundary, the associative division $\mathbb{R}$-algebra in a good triple can only be $\mathbb{C}$ and the homomorphism $\phi: \mathbb{Z}_{2} \rightarrow \operatorname{Aut}(\mathbb{C})$ must be given by $\phi_{g}=\kappa$. Now $\sigma$ should satisfy

$$
\sigma\left(g^{a}, g^{b}\right) \sigma\left(g^{a} g^{b}, g^{c}\right)=\omega\left(g^{a}, g^{b}, g^{c}\right) \phi_{g^{a}}\left(\sigma\left(g^{b}, g^{c}\right)\right) \sigma\left(g^{a}, g^{b} g^{c}\right),
$$

where $a, b, c \in\{0,1\}$. If one of $a, b, c$ is 0 , then the above equation holds automatically. Now let $a=b=c=1$. Then we have

$$
\sigma(g, g)=-\overline{\sigma(g, g)}
$$

This implies $\sigma(g, g) \in i \mathbb{R}$ where $i^{2}=-1$. By Lemma 4.5, we only need to take $\sigma(g, g)=i$ as any other choice will give an isomorphic ample division algebra in $\mathcal{C}\left(\mathbb{Z}_{2}, \omega, \mathbb{R}\right)$.

The division algebra corresponding to the triple $(\mathcal{C}, \phi, \sigma)$ is $A=\mathbb{C} \oplus \mathbb{C} u$ such that $u^{2}=i$. As an $\mathbb{R}$-space, it has basis $\{1, i, u, i u\}$. The multiplication of basis elements is given by the following table:

\begin{tabular}{|c|c|c|c|c|}
\hline & 1 & $i$ & $u$ & $i u$ \\
\hline 1 & 1 & $i$ & $u$ & $i u$ \\
\hline$i$ & $i$ & -1 & $i u$ & $-u$ \\
\hline$u$ & $u$ & $-i u$ & $i$ & 1 \\
\hline$i u$ & $i u$ & $u$ & -1 & $i$ \\
\hline
\end{tabular}


Remark 5.2. The nonassociative quaternions in the above proposition was discovered before by a completely different method, see [5]. Note that it is a fully division algebra, i.e., any nonzero (nonhomogeneous) element has a left and a right inverse. Let $a+b u$ be an arbitrary nonzero element with $a, b \in \mathbb{C}$. If $b=0$, then the inverse is $a^{-1}$. If $b \neq 0$, then

$$
(a+b u)\left(\frac{\bar{a}}{|a|^{2}-|b|^{2} i}-\frac{b}{|a|^{2}+|b|^{2} i} u\right)=1=\left(\frac{\bar{a}}{|a|^{2}-|b|^{2} i}-\frac{b}{|a|^{2}-|b|^{2} i} u\right)(a+b u) .
$$

Let $\mathbb{Z}_{2 n}=\left\langle g \mid g^{2 n}=\imath\right\rangle$ and consider the group homomorphism

$$
\phi: \mathbb{Z}_{2 n} \rightarrow \operatorname{Aut}(\mathbb{C}), \quad g^{r} \mapsto \begin{cases}\mathrm{Id}, & \text { if } r \text { is even; } \\ \kappa, & \text { if } r \text { is odd. }\end{cases}
$$

The following general formula for 3-cocycles on a cyclic group with coefficients in an algebraically closed field is well-known:

$$
\omega_{a}\left(g^{r}, g^{s}, g^{t}\right)=\zeta^{a\left[\frac{s+t}{2 n}\right] r}
$$

where $\zeta$ is a primitive $2 n$-th root of unity, $a \in[0,2 n-1]$ is an integer, and $[x]$ denotes the integer part of $x$, see e.g. [14]. Choose a nontrivial 3-cocycle $\omega_{a}$, i.e., $a \neq 0$. As the coefficients are in $\mathbb{R}^{*}$, there is only one choice, namely, $a=n$ and the 3-cocycle is given by

$$
\omega\left(g^{r}, g^{s}, g^{t}\right)=(-1)^{\left[\frac{s+t}{2 n}\right] r}
$$

If $(\mathbb{C}, \phi, \sigma)$ is a good triple in $\mathcal{C}\left(\mathbb{Z}_{2 n}, \omega, \mathbb{R}\right)$, then by (3.1) we have

$$
\sigma\left(g^{r}, g^{s}\right) \sigma\left(g^{r+s}, g^{t}\right)= \begin{cases}\sigma\left(g^{s}, g^{t}\right) \sigma\left(g^{r}, g^{s+t}\right), & \text { if } \mathrm{r} \text { is even; } \\ (-1)^{\left[\frac{s+t}{2 n}\right]} \frac{\sigma\left(g^{s}, g^{t}\right)}{\sigma}\left(g^{r}, g^{s+t}\right), & \text { if } \mathrm{r} \text { is odd. }\end{cases}
$$

Note that $\sigma$ takes values in $\mathbb{C}^{*}$ by the assumption on $\phi$ and (3.2). By (5.2), the restriction of $\sigma$ to the subgroup $\mathbb{Z}_{2 n}^{(2)}=\left\{g^{2 r} \mid 0 \leq r \leq n-1\right\}$ is a 2-cocycle. It is well-known that $\mathrm{H}^{2}\left(\mathbb{Z}_{2 n}^{(2)}, \mathbb{C}^{*}\right)=0$, hence we can set $\sigma\left(g^{r}, g^{s}\right)=1$ whenever $r$ and $s$ are even. It would be interesting to give a general solution for such $\sigma$. However, this seems to be out of reach for general $n$. In the following we give the result for $n=2$.

Proposition 5.3. Assume that $\sigma: \mathbb{Z}_{4} \times \mathbb{Z}_{4} \rightarrow \mathbb{C}^{*}$ satisfies (5.2) and $\sigma\left(l, g^{i}\right)=$ $\sigma\left(g^{i}, \imath\right)=\sigma\left(g^{2}, g^{2}\right)=1$ for $0 \leq i \leq 3$. Then $\sigma$ is determined by a pair of complex numbers $a=\sigma\left(g^{2}, g\right)$ and $b=\sigma\left(g^{3}, g\right)$ such that $(a b)^{2} \in i \mathbb{R}$. The values of $\sigma$ at other elements are

$$
\begin{gathered}
\sigma(g, g)=a b, \quad \sigma\left(g, g^{2}\right)=-\frac{\overline{a b}}{b}, \quad \sigma\left(g, g^{3}\right)=-\bar{b} \\
\sigma\left(g^{2}, g^{3}\right)=\frac{1}{a}, \quad \sigma\left(g^{3}, g^{2}\right)=\frac{\bar{b}}{\overline{a b}}, \quad \sigma\left(g^{3}, g^{3}\right)=-\frac{\bar{b}}{a} .
\end{gathered}
$$


The proof is straightforward, but tedious with computations. As mentioned above, the resulting division algebras can be viewed as some variations of the octonions.

Finally, we consider the Klein 4-group $K=\mathbb{Z}_{2} \times \mathbb{Z}_{2}=\left\langle g_{1}\right\rangle \times\left\langle g_{2}\right\rangle$. A general formula for 3-cocycles on $K$ is given in [14] as follows:

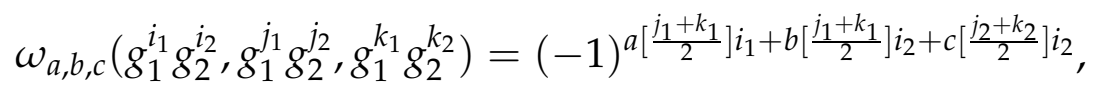

where $a, b, c, i_{1}, i_{2}, j_{1}, j_{2}, k_{1}, k_{2} \in\{0,1\}$. The only 3-coboundary is $\omega_{0,0,0}$. Let $\phi$ : $K \rightarrow \operatorname{Aut}(\mathbb{C})$ be the group homomorphism given by $g_{i} \mapsto \kappa, i=1,2$. If $(\mathbb{C}, \phi, \sigma)$ is a good triple in $\mathcal{C}\left(K, \omega_{a, b, c}, \mathbb{R}\right)$, then by (3.1) one has

$$
\begin{gathered}
\sigma\left(g_{1}^{i_{1}} g_{2}^{i_{2}}, g_{1}^{j_{1}} g_{2}^{j_{2}}\right) \sigma\left(g_{1}^{i_{1}+j_{1}} g_{2}^{i_{2}+j_{2}}, g_{1}^{k_{1}} g_{2}^{k_{2}}\right)= \\
(-1)^{a\left[\frac{j_{1}+k_{1}}{2}\right] i_{1}+b\left[\frac{j_{1}+k_{1}}{2}\right] i_{2}+c\left[\frac{j_{2}+k_{2}}{2}\right] i_{2}} \kappa^{i_{1}+i_{2}}\left(\sigma\left(g_{1}^{j_{1}} g_{2}^{j_{2}}, g_{1}^{k_{1}} g_{2}^{k_{2}}\right)\right) \sigma\left(g_{1}^{i_{1}} g_{2}^{i_{2}}, g_{1}^{j_{1}+k_{1}} g_{2}^{j_{2}+k_{2}}\right) .
\end{gathered}
$$

From this equation, it is easy to see that if $(a, b, c)=(1,1,1),(a, b, c)=(1,0,0)$, $(a, b, c)=(0,1,0)$, or $(a, b, c)=(0,0,1)$, one has

$$
\sigma\left(g_{1} g_{2}, g_{1} g_{2}\right)=-\sigma\left(g_{1} g_{2}, g_{1} g_{2}\right),
$$

hence $\sigma\left(g_{1} g_{2}, g_{1} g_{2}\right)=0$. This implies that there is no good triple in these cases.

In the following we consider the case $\omega=\omega_{1,0,1}$. For brevity, write

$$
\sigma\left(g_{1}, g_{2}\right)=x, \quad \sigma\left(g_{2}, g_{1}\right)=y \text {. }
$$

Applying (5.3) to the triple $\left(g_{1}, g_{1}, g_{1}\right)$, one has $\sigma\left(g_{1}, g_{1}\right)=-\overline{\sigma\left(g_{1}, g_{1}\right)}$. Hence, $\sigma\left(g_{1}, g_{1}\right) \in i \mathbb{R}$. Similarly, one has $\sigma\left(g_{2}, g_{2}\right) \in i \mathbb{R}$. Assume that

$$
\sigma\left(g_{1}, g_{1}\right)=p i, \quad \sigma\left(g_{2}, g_{2}\right)=q i,
$$

where $p, q \in \mathbb{R}^{*}$. Now applying (5.3) to the triple $\left(g_{1}, g_{1}, g_{2}\right)$, one gets

$$
\sigma\left(g_{1}, g_{1}\right)=\overline{\sigma\left(g_{1}, g_{2}\right)} \sigma\left(g_{1}, g_{1} g_{2}\right) .
$$

This implies $\sigma\left(g_{1}, g_{1} g_{2}\right)=\frac{p i}{\bar{x}}$. Again, applying (5.3) to the triple $\left(g_{1} g_{2}, g_{1}, g_{1}\right)$, one obtains $\sigma\left(g_{1} g_{2}, g_{1}\right) \sigma\left(g_{2}, g_{1}\right)=-\sigma\left(g_{1}, g_{1}\right)$ and $\sigma\left(g_{1} g_{2}, g_{1}\right)=\frac{-p i}{y}$. Finally one applies (5.3) to the triple $\left(g_{1}, g_{2}, g_{1}\right)$, and obtains

$$
\sigma\left(g_{1}, g_{2}\right) \sigma\left(g_{1} g_{2}, g_{1}\right)=\overline{\sigma\left(g_{2}, g_{1}\right)} \sigma\left(g_{1}, g_{1} g_{2}\right) .
$$

It follows that $x \frac{-p i}{y}=\bar{y} \overline{\bar{x}}$, hence $x \bar{x}+y \bar{y}=0$ and $x=y=0$. That is absurd. Therefore, there is no good triple, hence no ample division algebra in this case either. Similarly, one has the same conclusion for $\omega_{1,1,0}$ and $\omega_{0,1,1}$.

For other group homomorphisms $\phi: K \rightarrow \operatorname{Aut}(\mathbb{C})$ such that $\phi(G)=\{\operatorname{Id}, \kappa\}$, we have the same conclusion via a similar procedure as above.

Proposition 5.4. If $\omega$ is not a coboundary, then there is no ample division algebra in $\mathcal{C}(K, \omega, \mathbb{R})$.

Acknowledgements: The research of Huang was partially supported by the SDNSF grant 2009ZRA01128 and the IIFSDU grant 2010TS021. The paper was written while Huang was visiting the University of Antwerp and he is very grateful for its hospitality. He acknowledges the Belgian FWO for the financial support which makes the visit possible. 


\section{References}

[1] Akrami, Seyed Ebrahim; Majid, Shahn: Braided cyclic cocycles and nonassociative geometry. J. Math. Phys. 45 (2004), no. 10, 3883-3911.

[2] Albuquerque, Helena; Majid, Shahn: Quasialgebra structure of the octonions. J. Algebra 220 (1999), no. 1, 188-224.

[3] Albuquerque, Helena; Elduque, Alberto; Pérez-Izquierdo, José María: $\mathrm{Z}_{2}$-quasialgebras. Comm. Algebra 30 (2002), no. 5, 2161-2174.

[4] Albuquerque, Helena; Santana, Ana Paula: A note on quasiassociative algebras. The J. A. Pereira da Silva birthday schrift, 5-16, Textos Mat. Sér. B, 32, Univ. Coimbra, Coimbra, 2002.

[5] Althoen, S. C.; Hansen, K. D.; Kugler, L. D.: A survey of four-dimensional C-associative algebras. Algebras Groups Geom. 21 (2004), no. 1, 9-27.

[6] Baez, John C.: The octonions. Bull. Amer. Math. Soc. (N.S.) 39 (2002), no. 2, 145-205.

[7] Baez, John C.: Division algebras and quantum theory. Foundations of Physics 42 (2012), no. 7, 819-855.

[8] Calaque, Damien; Etingof, Pavel: Lectures on tensor categories. in: Quantum groups, 1-38, IRMA Lect. Math. Theor. Phys., 12, Eur. Math. Soc., Zürich, 2008.

[9] Conway, John H.; Smith, Derek A.: On quaternions and octonions: their geometry, arithmetic, and symmetry. A K Peters, Ltd., Natick, MA, 2003.

[10] Dijkgraaf, Robbert; Pasquier, Vincent; Roche, Philippe: Quasi Hopf algebras, group cohomology and orbifold models. in: Recent Advances in Field Theory, Annecy-le-Vieux, 1990, Nucl. Phys. B Proc. Suppl. 18B (1990) 60-72.

[11] Dyson, Freeman J.: The threefold way. Algebraic structure of symmetry groups and ensembles in quantum mechanics. J. Mathematical Phys. 31962 1199-1215.

[12] Elduque, Alberto: Gradings on octonions. J. Algebra 207 (1998), no. 1, 342-354.

[13] Hoàng Xuân Sính: Gr-catégories. Université Paris VII, Thèse de doctorat (1975). available at http:/ / www.math.rwth-aachen.de/ kuenzer/sinh.html.

[14] Huang, Hua-Lin; Liu, Gongxiang; Ye, Yu: The braided monoidal structures on a class of linear Gr-categories. Algbr. Represent. Theory 17 (2014), no. 4, 12491265.

[15] Joyal, André; Street, Ross: Braided tensor categories. Adv. Math. 102 (1993), no. $1,20-78$.

[16] Karrer, Guido: Graded division algebras. Math. Z. 133 (1973), 67-73. 
[17] Lam, Tsit Yuen: The algebraic theory of quadratic forms. Mathematics Lecture Note Series. W. A. Benjamin, Inc., Reading, Mass., 1973.

[18] Majid, Shahn: Foundations of quantum group theory. Cambridge University Press, Cambridge, 1995.

[19] Ostrik, Victor: Module categories, weak Hopf algebras and modular invariants. Transform. Groups 8 (2003), no. 2, 177-206.

[20] Van Oystaeyen, Fred; Zhang, Yinhuo: The Brauer group of a braided monoidal category. J. Algebra 202 (1998), no. 1, 96-128.

School of Mathematics, Shandong University,

Jinan 250100, China

email:hualin@sdu.edu.cn

Department of Mathematics and Computer Science,

University of Antwerp,

Middelheimlaan 1,

B-2020 Antwerp, Belgium

email:fred.vanoystaeyen@ua.ac.be

Department of Mathematics and Statistics,

University of Hasselt,

Universitaire Campus,

3590 Diepenbeek, Belgium

email:yinhuo.zhang@uhasselt.be 